\title{
GCU
}

Glasgow Caledonian

University

University for the Common Good

\section{Mobility prediction for traffic offloading in cloud cooperated mmWave 5G networks}

\author{
Liarokapis, Dimitrios
}

Published in:

2017 9th IEEE-GCC Conference and Exhibition (GCCCE)

DOI:

10.1109/IEEEGCC.2017.8448090

Publication date:

2018

Document Version

Author accepted manuscript

Link to publication in ResearchOnline

Citation for published version (Harvard):

Liarokapis, D 2018, Mobility prediction for traffic offloading in cloud cooperated mmWave 5G networks. in 2017 9th IEEE-GCC Conference and Exhibition (GCCCE). IEEE, IEEE-GCC, Manama, Bahrain, 8/05/17.

https://doi.org/10.1109/IEEEGCC.2017.8448090

\section{General rights}

Copyright and moral rights for the publications made accessible in the public portal are retained by the authors and/or other copyright owners and it is a condition of accessing publications that users recognise and abide by the legal requirements associated with these rights.

Take down policy

If you believe that this document breaches copyright please view our takedown policy at https://edshare.gcu.ac.uk/id/eprint/5179 for details of how to contact us. 


\title{
Mobility Prediction for Traffic Offloading in Cloud Cooperated mmWave 5G Networks
}

\author{
Dimitrios Liarokapis \\ School of Engineering and Built Environment \\ Glasgow Caledonian University \\ United Kingdom \\ Dimitrios.Liarokapis@gcu.ac.uk \\ 1.dhmhtrhs@gmail.com
}

\begin{abstract}
Future cellular networks are predicted to witness an extraordinary increase in mobile related traffic load in the next 10 years. This is the catalyst for the creation of the 5th generation (5G) cellular networks that could potentially accommodate much higher data rates by a factor of 1,000. Currently, there have been quite a few different proposed architectures that promise to support such an overwhelming demand. The utilization of the ultrawideband aspect of the mmWave bands is considered at the moment one of the most promising approaches, since it makes use of very high frequencies and therefore it offers a much higher theoretical channel capacity for data transfer. Under the umbrella of mmWave bands to be used for the implementation of $5 G$ networks, many studies have proposed the incorporation of the currently dominant 4G/LTE technology to function alongside $5 G$ and to be solely responsible for signaling and control data transfers (C-Plane), so as user data (U-Plane) will be given priority over higher $5 G$ data rates whenever and wherever available. This heterogeneous network that could operate in a range of different frequencies over the same area and at the same time, may be enhanced even further with the use of a cloud infrastructure for radio access network $(C$ $R A N)$ that would be responsible for overseeing the entire network topology's optimized functionality. Such a complex architecture is certain to bring to the surface some very challenging problems. The switching between $4 G$ and $5 G$, whenever a User Equipment (UE) exits a pico cell or enters a new pico cell, is not as simple as normal handovers between cells that operate under the same technology. Service break ups and disruption of service are only two of the devastating results in user experience when dealing with sudden handovers between technologies and not just cells. In this paper, a mobility prediction scheme is proposed that makes use of C-RAN, titled Cloud Cooperated Mobility Prediction (CCMP) and instructs UEs under a certain probability whether or not they are predicted to exit a pico cell in the near future. If there is a positive chance for this to happen, the UE will take all the necessary actions to offload its data traffic from the U-Plane to the C-Plane in a much smoother and more efficient way.
\end{abstract}

Keywords- 5G; mmWave; heterogeneous network; cloud radio access network; mobility prediction; traffic offloading; CCMP

\section{INTRODUCTION}

The way users take advantage of mobile user equipment with internet connection capabilities, such as smart phones and tablets, has resulted in a dramatic increase of internet related traffic loads in the last 5 years. In addition, the demand for more IP data to be transferred will be given an even stronger boost, since novel computing technologies are emerging and will become dominant in the near future. Some of these include the Internet of Everything (IoE), Machine to Machine (M2M) communications, Vehicular Networks and many more. This extreme increase in the demand for data transfer will require the improvement of system data rates of 1,000 fold in the next 10 years.

Currently, there are 4 different proposed architectures for $5 \mathrm{G}$ to follow, that are explored in the literature [1], [2] and ambitiously aim to implement an appropriate solution to the overwhelming demand for fast data transmission. These are briefly presented below.

a) Enhanced MIMO (Multiple-Input Multiple-Output): multi-layer spatial multiplexing techniques in order to improve spectral efficiency.

b) CoMP (Cooperative Multi-Point Transmission): basestation cooperation for the improvement of number of outages at the outer areas of the cells via inter cell interference alleviation.

c) Heterogeneous Networks: system rate improvement by offloading traffic from macro base stations (BSs) to pico or femto BSs placed inside the macro BS.

d) Bandwidth Expansion: system rate improvement by aggregating pieces of spectrum in microwave bands.

CCMP bases its functionality, as it will be presented in detail later on, on the architectures proposed in $c$ ) and $d$ ) with some added operational elements presented in $b$ ). As a result, a cloud cooperated heterogeneous network (C-HetNet) [3] is created. Smaller transmission range pico basestations operate under the coverage of wider transmission range macro basestations, while all of these BSs have access to the same cloud radio access network [4] for centralized overall control and management. The heterogeneous aspect of such an approach lays on the use of different frequencies for the same geographical area; low frequencies for the macro basestations (e.g. $2 \mathrm{GHz}$ ) and higher frequencies for the pico basestations (e.g. $3 \mathrm{GHz}$ or $60 \mathrm{GHz}$ ) [5].

As long as a UE is positioned appropriately, it may use $4 \mathrm{G}$ for control data (C-Plane) and 5G for the more demanding user data (U-Plane). Since pico cells will not be able to cover the 
macro cell $100 \%$ there will be areas inside the macro cell where only $4 \mathrm{G}$ coverage will be available. In cases where UEs are highly mobile, there are high chances that they might migrate from a pico cell to an area in the macro cell where there is no 5G coverage. Currently, soft handovers between macro BSs are possible, as a UE does not require to disconnect from BS1 before it connect to BS2, as there usually is a small overlap in coverage between neighbouring BSs. This is not the case for the type of HetNets described above. The UE will be forced to perform a hard handover in order to switch from $5 \mathrm{G}$ to $4 \mathrm{G}$ for data traffic as the exit from the pico cell may happen without warning. Such a hard handover is a much more complex procedure as there cannot be any transition time from one connectivity state to the next, especially under the extreme characteristics introduced by mmWave communication [6].

In this paper, a novel $5 \mathrm{G}$ cellular network scheme titled Cloud Cooperated Mobility Prediction (CCMP) is proposed, where UEs, with the help of the cloud infrastructure, predict their movement and the possibility of exiting a pico cell's coverage area in the near future. The CCMP's main entity operating in the cloud, is able to calculate a certain probability for every individual UE under which the UE will initiate a handover for the data traffic from the U-Plane to the C-Plane while it is still placed inside the pico cell. Therefore, connectivity loss for data traffic is minimized by running a soft instead of a hard handover between pico and macro BSs cells.

The remainder of this paper is organized as follows: Section II presents in detail relevant literature review on the CHetNet architecture that makes use of a cloud radio access network and UE mobility issues. Section III describes the proposed novel CCMP scheme and analyzes its advantages compared to conventional approaches. Future work and concluding remarks are presented in Section IV.

\section{LITERATURE REVIEW}

C-HetNets are constructed by making use of two very popular and quite promising cellular network technologies. Those are a) multi-band heterogeneous networks (multi-band HetNets) and b) cloud radio access networks (C-RAN). In addition, since mobility is the dominant factor for the execution of handovers, relevant issues need to be addressed.

\section{A. Multi-band HetNets}

A multi-band HetNet is a cellular network that operates on multiple frequency bands at the same time and for overlapping geographical areas. Conventional BSs (macro BSs) cover a large area, parts of which may also be covered by low power small coverage area BSs (pico BSs). Since different frequency bands are used by the macro and pico BSs, there is no need for interference mitigation techniques [7].

\section{B. Cloud Radio Access Network}

The concept of C-RAN was first introduced in [4]. Its goal was to address the challenges mobile operators are facing with regards to their radio access networks, such as cost, energy consumption, spectral efficiency, smooth evolution via an open platform for multiple standards support and additional revenue generating services.
C-RAN was proposed to operate following two different methods that consequently offer two separate architectures. The first is a fully centralized solution, where baseband processing takes place centrally for all BSs and the second is a partially centralized solution where baseband processing takes place in a distributed manner and closer to the BSs. By adopting any of the two available architectures of C-RAN, mobile operators may enjoy multiple benefits but also face important technical challenges.

Advantages include a) energy efficiency/green infrastructure, b) cost saving, c) capacity improvement, d) adaptability to non-uniform traffic and e) smart traffic offloading. On the other hand, mobile operators need to address technical challenges such as a) radio under low cost optical network, b) advanced cooperative transmission and reception, c) baseband pool interconnection, d) basestation virtualization and e) service on edge.

\section{C-HetNets}

The outcome of combining together HetNets and a Cloud Radio Access Network is the Cloud Cooperated Heterogeneous Network as it is described in [3]. One of the main challenges introduced with multi-band heterogeneous networks is the fact that UEs are required to operate in a multi-band mode as they need to be able to connect to different BSs, transmitting in different frequencies at the same time. Running cell searches for pico BSs quite frequently while connected with a macro BS is extremely power consuming and introduces unwanted overall delay. Furthermore, effective planning for the placement of pico BSs becomes a challenge. Since pico BSs operate in very high frequencies (e.g. $60 \mathrm{GHz}$ ) path loss is affected by the distance between the UE and the BS with very negative outcomes.

A promising solution to the above issues is the use of CHetNets. According to this architecture, all pico and macro BSs connect to the same cloud radio access network. As a result, the control of all pico BSs is now laid upon the C-RAN of the $\mathrm{C}$-HetNet and is maintained via information provided by the macro BS. As an operating requirement, this approach specifies the splitting of user and control data (U/C splitting). All control traffic between UEs and the network is forwarded to the C-RAN via the macro BS and thus it manages the Cplane for all users. On the other hand, all user traffic (U-plane) is forwarded to the network via the pico BS associated with the particular UE. As a result, the macro BS supports the UE to successfully perform cell searches, handovers and user associations and re-associations with regards to pico BS connectivity.

\section{Mobility in $5 G$ Cellular Networks}

Mobility management in cellular technologies is considered to be a vital operation for the delivery of uninterrupted service to users as they move from cell to cell. Dealing with a single set of operational bands, which is the norm for current cellular technologies ( $3 \mathrm{G}$ and $4 \mathrm{G}$ ), makes things much less complex. When a UE senses the existence of a new cell that overlaps with its existing associated cell, then it may transfer all of its communication channels from one BS to another without losing connectivity. 
Since the dominant proposed $5 \mathrm{G}$ architectures result in the creation of heterogeneous topologies, since different frequency bands overlap in the same geographical area, handovers are now of a different nature. For the completion of a handover, switching between technologies and not just BSs is required. The main concept behind these handovers and in order to have an uninterrupted service, is mobility prediction. Several studies propose different mobility prediction schemes that are based on UEs' mobility history [8]-[10]. The main disadvantage of such schemes is the fact that they make use of "old" information (mobility history) in order to predict future movement. Indeed, mobility history is an obvious candidate to play a key role in predicting future movement, but the duration of past movement considered, should be kept as short as possible in order to increase the confidence level of the schemes' outcomes.

\section{ClOUd COOPERATED MobILITy PREDICTION (CCMP)}

In this paper, a Cloud Cooperated Mobility Prediction (CCMP) scheme for traffic offloading in $5 \mathrm{G}$ cellular networks is proposed. The basic concept of CCMP is to allow UEs to determine whether or not they consider themselves to be highly mobile or not and therefore predict if they will remain within a pico BS's transmission range for an upcoming time interval. This decision will be based on the execution of a mobility prediction algorithm that requires as input, information derived from all other UEs based inside the same pico base station's transmission range boundaries. UEs will be able to access information regarding their neighbouring UEs via an established cloud infrastructure. In case a UE determines that it is highly mobile and predicts that it will move out of the current BS's range, then it must take action in order to offload its data traffic from the U-Plane and 5G to the C-Plane and 4G. If not, the UE will maintain its default status that dictates the use of the U-Plane for data traffic and the use of the C-Plane for control traffic.

One of CCMP's main functioning requirements is the use of time slots of any value between 5 and 10 seconds. This value has to be fixed for all UEs in the topology and can be set individually by each base station according to how dynamic or static its coverage area is. Intuitively, it can be argued that a shorter in duration time slot should be set for highly dynamic topologies, as changes are more probable to take place and therefore they have to be documented accurately. At the end of every time slot, a UE should calculate its mobility level and decide whether to offload its data traffic to the C-Plane or not. Instead of introducing a single formula that would be used by the UEs to predict their near future BS association, three different rules need to be applied that are created based on the answers to the following questions:

- What parameters are generally responsible for significantly altering the network's topology?

- What parameters make a cellular network to be considered as highly dynamic or static?

- What parameters are responsible for causing a change to the number of UEs within a base station's coverage area?
As a result, it is concluded that the following parameters should be considered:

- UEs' speed and distance from the pico BS.

- Local UE density within the pico BS's coverage area.

- UEs' trajectories.

CCMP aims to enhance the network's overall performance by allowing UEs to determine under what technology they will exchange data traffic. The following pattern needs to be addressed by all three rules:

- If a UE predicts that it is highly probable to exit its current cell in the next time slot, it will act proactively and offload its data traffic from the U-Plane to the CPlane based on a high value, fixed probability. Thereafter, it is responsible to continuously monitor any changes in the value of the above mentioned probability and in case it reaches a low enough level, it will reassociate with a pico BS for future data traffic transmission.

- If a UE predicts that it is not probable to exit its current cell in the near future, it will most probably maintain its default connectivity status and will continue monitoring the value of this probability for future reference.

\section{A. Speed and Distance (Rule 1)}

Only a few seconds before the end of every time slot, all UEs need to report their current speed at that time and their distance from the pico BS to the cloud infrastructure. The distance can be calculated by measuring the power of the received signal at the $\mathrm{BS}$. The CCMP module operating in the cloud element of the network, after having all the speeds from all the UEs associated with every pico BS, will calculate part of the CCMP probability $\left(\mathrm{P}\left(\mathrm{u}_{\mathrm{n}}, \mathrm{d}_{\mathrm{n}}\right)\right)$ according to Rule 1 for every individual UE and will send all corresponding packets with the appropriate information. The following formula will be used for the calculation of $\mathrm{P}\left(\mathrm{u}_{\mathrm{n}}, \mathrm{d}_{\mathrm{n}}\right)$.

$$
\mathrm{P}\left(\mathrm{u}_{\mathrm{n}}, \mathrm{d}_{\mathrm{n}}\right)=\left\{\begin{array}{c}
\frac{\mathrm{T}_{\mathrm{R}} \cdot\left|\mathrm{u}_{\mathrm{n}}\right|}{\max \left(\left|\mathrm{u}_{1}\right|,\left|\mathrm{u}_{2}\right|, \ldots, \mid \mathrm{u}_{\mathrm{n}}\right) \cdot\left(2 \mathrm{~T}_{\mathrm{R}}-\mathrm{d}_{\mathrm{n}}\right)} \text { when } \mathrm{u}_{1}, \mathrm{u}_{2}, \ldots, \mathrm{u}_{3} \neq 0 \\
0, \text { when } \mathrm{u}_{1}, \mathrm{u}_{2}, \ldots, \mathrm{u}_{3}=0
\end{array}\right.
$$

where $\mathrm{T}_{\mathrm{R}}$ : the pico BS's transmission range,

$\mathrm{u}_{\mathrm{n}}$ : the neighbouring node's speed,

$\mathrm{d}_{\mathrm{n}}$ : the distance between the originating and the neighbouring node and

$$
0 \leq \mathrm{P}\left(\mathrm{u}_{\mathrm{n}}, \mathrm{d}_{\mathrm{n}}\right) \leq 1 .
$$

Let us consider the scenario where CCMP makes use of only formula (1) to calculate the offloading probability for every individual UE. If the specific UE or all UEs are completely static, then this UE or all UEs do not cause any additional overhead and thus they should be continuing to benefit from high, $5 \mathrm{G}$ data rates. Truly, according to (1), $\mathrm{P}\left(\mathrm{u}_{\mathrm{n}}\right.$, $\mathrm{d}_{\mathrm{n}}$ ) is equal to 0 and therefore the UE will not attempt to offload its data traffic to the C-Plane. The opposite side of the 


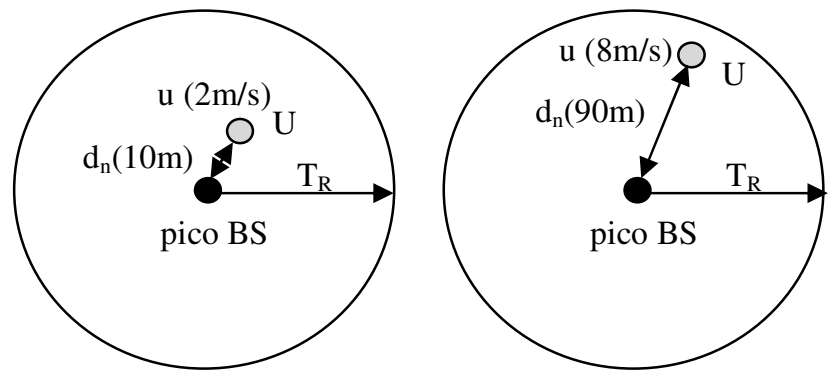

Fig. 1. CCMP Rule 1.

extreme is for a UE to have the maximum value of speed compared to all other neighbouring UEs and to be placed at the edge of the cell. Intuitively, it can be argued that most likely this UE will exit its current cell in the very near future and therefore, it should proactively disconnect from the pico base station. In this case, according to $(1), P\left(u_{n}, d_{n}\right)$ is equal to 1 and therefore the UE will be forced to offload its data traffic to the C-Plane.

For all other cases, not covered by the two extremes described above, the lower the UE's absolute speed value, the lower the probability for data traffic offloading. In addition, the further the UE is placed form the pico BS, the highest the probability to offload the data traffic. For example and as shown in Figure 1, if the pico cell's transmission range is $100 \mathrm{~m}$, the maximum UE's speed is $10 \mathrm{~m} / \mathrm{s}$ and the UE is placed $10 \mathrm{~m}$ away from the pico BS with a speed of $2 \mathrm{~m} / \mathrm{s}$ the probability to offload its data traffic is equal to $10.53 \%$. If the UE is placed $90 \mathrm{~m}$ away with a speed of $8 \mathrm{~m} / \mathrm{s}$ the probability is equal to $72.73 \%$. As a general outcome derived from (1) it can be stated that nodes with low speeds and close to the pico BS are much less probable to have an alteration on their connectivity status compared to UEs with high speeds placed far away from the pico BS.

\section{B. Local Density (Rule 2)}

The end result of the comparison between a pico BS's local density (y) and the macro BS's local density (v) could be a deciding factor when considering the calculation of the applied probability for UEs to offload their data traffic. These two densities are defined below:

$$
\begin{gathered}
y=\frac{\text { Number of pico Neighbouring UEs }}{\text { Pico BS Transmission Area }}=\frac{\mathrm{n}_{\text {pico }}}{\pi \cdot \mathrm{T}_{\text {picoR }}{ }^{2}}, \\
\mathrm{v}=\frac{\text { Number of macro Neighbouring UEs }}{\text { Macro BS Transmission Area }}=\frac{\mathrm{n}_{\text {macro }}}{\pi \cdot \mathrm{T}_{\text {macroR }}{ }^{2}} .
\end{gathered}
$$

Let us consider the following two extreme network conditions where for scenario 1 the micro BS's local density is much higher than the macro BS's local density $(y>>v)$ and for scenario 2 we set $y<<v$. These scenarios are reflected in Figure 2 .

CCMP's goal under Rule 2 is to successfully predict the difference in density locally inside the pico BS's transmission area at the beginning of the next time slot. In case of scenario 1 , it is most probable that many pico associated UEs will travel
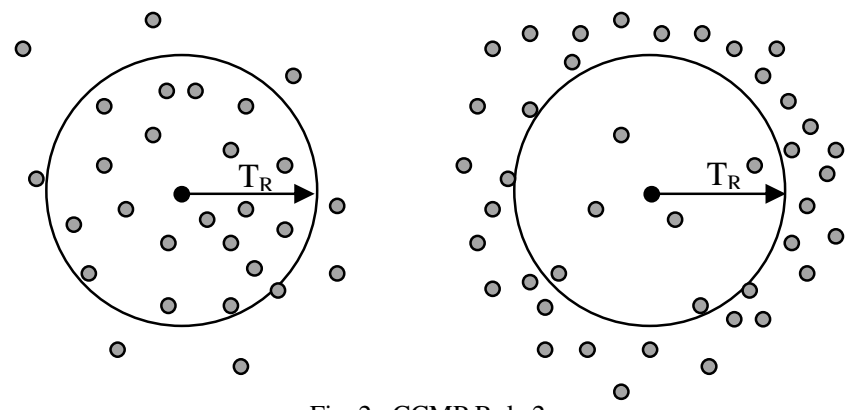

Fig. 2. CCMP Rule 2.

outside the pico BS's transmission area compared to the possible number of macro UEs passing inside the pico transmission area. In turn, for scenario 2 , it is most probable that the number of macro UE's added in the pico BS's list of associated UEs at the beginning of the next time slot, will be much larger than the pico UEs that may travel outside the pico transmission area. Despite the fact that for both scenarios it is clear that the pico cell's local density will greatly change (decreased for scenario 1 and increased for scenario 2), the calculation of the offloading probability must follow separate paths. For scenario 1, since the pico BS's transmission area is predicted to have fewer associated UEs during the next time slot, none of these UEs should offload their data traffic to the C-Plane as their decreased number will ease the traffic transmission burden by itself. For scenario 2, due to the prediction that the pico transmission area will most likely have an increased number of associated UEs in the near future, it must set the offloading probability for currently associated UEs to a high value in order for the pico BS to be able to satisfy the increase demand for high data rates from a high number of UEs.

There is one more scenario that needs consideration. What will be the degree of change in a pico BS's local density when $\mathrm{y} \approx \mathrm{v}$ ? Leaving UEs' speeds and trajectories out of the equation as they are considered by other rules, it is intuitively predicted that it is most probable that the number of UEs leaving the pico transmission area is very close to the number of UEs coming into the pico transmission area. Thus, no significant change occurs with regards to local density and consequently the value of the offloading probability should be set to a low value, since the overall pico BS's load is predicted to remain almost unchanged for the duration of the next time slot.

The offloading probability based on Rule 2 is calculated using the below formula:

$$
P(y, v)=\left\{\begin{array}{l}
\frac{v}{y}, y>v \\
1-\frac{y}{v}, y \leq v
\end{array}\right.
$$

The closer $\mathrm{P}(\mathrm{y}, \mathrm{v})$ is to 1 the higher the chance of an increased number of future associated UEs, therefore the overall offloading probability should also be high. When $\mathrm{P}(\mathrm{y}$, v) is calculated close or equal to 0 that is the outcome to the fact that no considerable changes in the number of associated UEs will take place in the next time slot, therefore the overall offloading probability should be assigned a low value. 


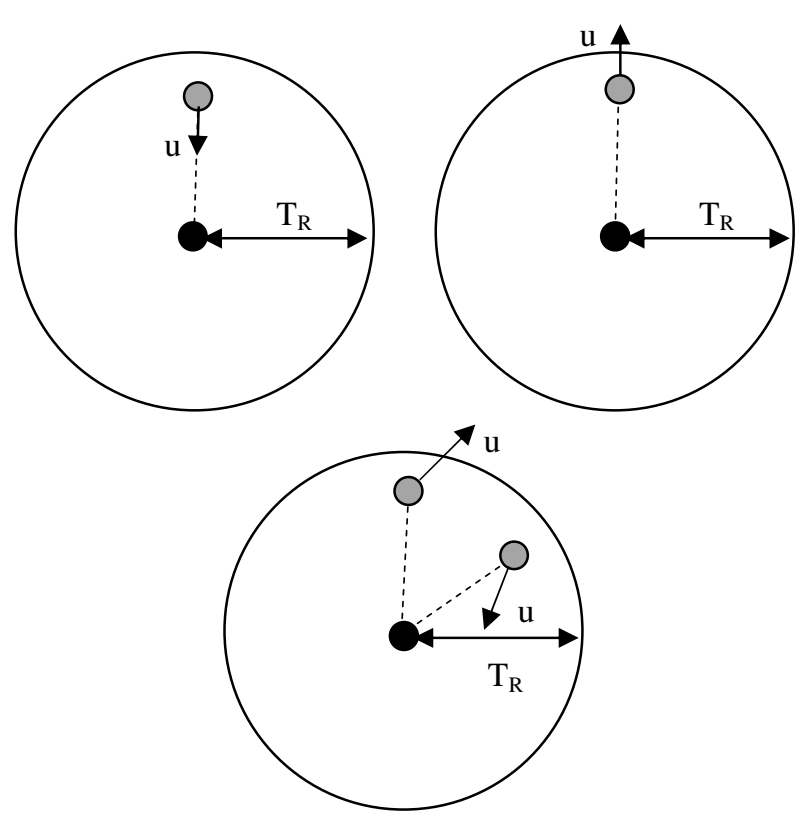

Fig. 3. CCMP Rule 3.

\section{Trajectories (Rule 3)}

Let us consider the scenario where a UE is moving towards the pico BS. If the speed factor is excluded from the affecting variables, as it is analyzed in a previous section, it can be concluded that there is a low probability for this UE to exit the pico transmission area in the next time slot as shown in Figure 3 . In case the UE moves in an opposite direction and away from the pico BS, the probability of the UE exiting the transmission area is much greater as it can be seen in Figure 3. In general we define as $\mathrm{z}$ the angle between the UE's trajectory and the distance vertex between UE and pico BS. For the first scenario $\mathrm{z}=0^{\circ}$ whereas for the second scenario $\mathrm{z}=180^{\circ}$.

As shown in Figure 3, it is argued that the degree of change in the transmission area of a pico BS starting from 0 for $\mathrm{z}=0^{\circ}$ constantly increases and reaches 1 for $\mathrm{z}=180^{\circ}$. The UE's offloading probability also needs to follow a similar pattern, so as UEs movE towards the pico BS, they should have a greater chance of maintaining their connectivity status $(\mathrm{P}(\mathrm{z})$ closer to $0)$ compared to the UEs moving away from the pico BS $(\mathrm{P}(\mathrm{z})$ closer to 1). The formula to calculate the offloading probability $\mathrm{P}(\mathrm{z})$ for a specific UE under Rule 3 is given below:

$$
\mathrm{P}(\mathrm{z})=\left\{\begin{array}{c}
\frac{\sin \mathrm{z}}{2}, \text { where } 0^{\circ} \leq \mathrm{z} \leq 90^{\circ} \\
\frac{1-\cos \mathrm{z}}{2}, \text { where } 90^{\circ}<\mathrm{z} \leq 180^{\circ}
\end{array}\right.
$$

The limitation of this method is the overwhelming degree of complexity for the calculation of the angle $\mathrm{z}$, resulting in making this rule unusable. Our original goal for Rule 3 was to determine whether a specific UE moves towards to or away from the pico BS. This goal could also be achieved by comparing the distances between pico BS and UE at two different points in time, such as the beginning and end of the previous time slot. As a result, Rule 3 could be substituted by Rule $3 b$ as shown in Figure 4.
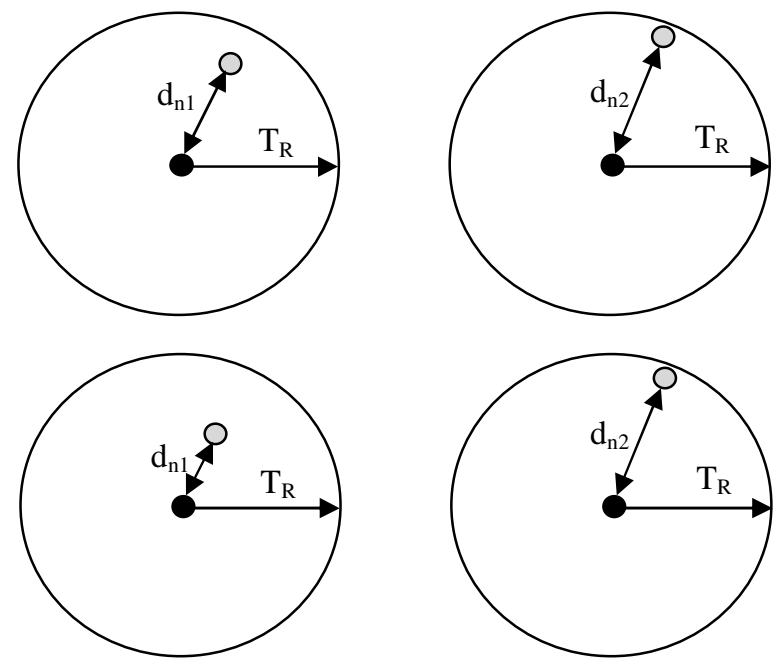

Fig. 4. CCMP Rule 3b.

\section{Difference in Distances (Rule $3 b$ )}

Let us define that $d_{1}$ is the distance between the pico BS and the UE at the start of the previous time slot and that $d_{2}$ is the distance between the same network entities at the end of the previous tie slot. Using the following formula, we are able to calculate what is the value of the offloading probability $\mathrm{P}\left(\mathrm{d}_{1}\right.$, $\mathrm{d}_{2}$ ) for this UE at the start of the next time slot.

$$
\mathrm{P}\left(\mathrm{d}_{1}, \mathrm{~d}_{2}\right)=\left\{\begin{array}{c}
\frac{\left|\mathrm{d}_{1}-\mathrm{d}_{2}\right|}{\mathrm{T}_{\mathrm{R}}}=\frac{|\Delta \mathrm{d}|}{\mathrm{T}_{\mathrm{R}}} \text {, when } \mathrm{d}_{1} \leq \mathrm{d}_{2} \\
0, \text { when } \mathrm{d}_{1}>\mathrm{d}_{2}
\end{array}\right.
$$

For all the cases where $d_{1} \leq d_{2}$, that is true when the UE moves away from the pico BS, the closer $\mathrm{P}\left(\mathrm{d}_{1}, \mathrm{~d}_{2}\right)$ is to 0 , which is the case when the UE is quite static, the lower the probability should be to offload its data traffic to the C-Plane, as the chance for the UE to exit the pico transmission area in the next time slot is extremely low. This is the case when the UE's distances from the pico BS at the beginning and end of the previous time slot are similar in value. This is also true even if the UE stands at the boundary of the pico transmission area. The opposite extreme takes place if at the beginning, the UE is placed next to the BS and at the end it is placed at the boundary of the transmission area. Therefore, $\Delta \mathrm{d}$ is equal to TR and from (4) it is derived that the offloading probability for the UE should be set to 1 , forcing it to fully associate with the macro BS for both control and data transfers. For all the cases where $d_{1}>d_{2}$, that is true when the UE moves towards the pico $\mathrm{BS}$, then the offloading probability is set to 0 , as the UE is considered to move on a path that cannot lead outside the pico transmission area. Bear in mind, that UEs' speeds are not considered in this rule and therefore all symmetries that may occur due to extremely high speeds, are excluded from our algorithmic design.

\section{E. Final Offload Probability Calculation}

After a UE has received the specific values of the three different offloading probabilities that are derived from the three rules and calculated by the cloud infrastructure element of CCMP, the UE needs to calculate the value of the final 
offloading probability P. How will the final probability value be calculated before it is used by the UE? Before answering this question, an answer first needs to be given to the following question. What rule of the three has the largest priority? Is there a rule that could better represent A UE's chances of leaving the pico transmission area in the next time slot? If yes, that rule should be favoured against the other two. Priority between rules could be represented by a percentage weight. For example, there could be the case where 0.5 priority is given to Rule $1,0.3$ priority is given to Rule 2 and finally 0.2 priority to Rule 3b. Apparently, the sum of all priority weights should result to 1 . The following formula should be used for the calculation of the final value of $P$.

$$
\mathrm{P}=\mathrm{k}_{1} \cdot \mathrm{P}\left(\mathrm{u}_{\mathrm{n}}, \mathrm{d}_{\mathrm{n}}\right)+\mathrm{k}_{2} \cdot \mathrm{P}(\mathrm{y}, \mathrm{v})+\mathrm{k}_{3} \cdot \mathrm{P}\left(\mathrm{d}_{1}, \mathrm{~d}_{2}\right)
$$

where $\mathrm{k}_{1}+\mathrm{k}_{2}+\mathrm{k}_{3}=1$.

There is no clear indication at the moment to support inequality amongst the three rules. Thus, it is assumed that all rules represent the network's dynamics equally well, as all factors advantaged by these rules (speed - distance, local density and distance difference) equally affect the prediction of UEs' movement. Therefore, formula (5) is defined as follows.

$$
\mathrm{P}=\frac{1}{3} \cdot\left(\mathrm{P}\left(\mathrm{u}_{\mathrm{n}}, \mathrm{d}_{\mathrm{n}}\right)+\mathrm{P}(\mathrm{y}, \mathrm{v})+\mathrm{P}\left(\mathrm{d}_{1}, \mathrm{~d}_{2}\right)\right)
$$

\section{CONCLUSION \& FUTURE WORK}

Concluding remarks and future work are presented in this section.

\section{A. Conclusion}

In this paper a Cloud Cooperated Mobility Prediction (CCMP) scheme for 5G cellular networks was proposed to eliminate service interruption for handovers between different technologies and thus to increase overall network performance and user satisfaction. CCMP predicts the mobility level of UEs and by combining 3 mobility prediction rules it calculates a certain probability for individual UEs to use in order to decide whether or not they will exit a pico transmission area in the upcoming time slot. Low probability values are set for UEs that determine it is not likely to exit the pico cell and therefore it is unlike to offload their data transmission from $5 \mathrm{G}$ and the UPlane to $4 \mathrm{G}$ and C-Plane. High probability values are set for UEs that predict exiting from the pico cell and therefore smoothly offload their user data transmission to the C-Plane before disconnecting from the pico BS.

In order for CCMP to predict any of the mobile UEs' future movement, it incorporates the use of 3 rules that make use of the UEs' speed, distance from the pico BS, difference in distance between time slots and difference between local and overall topology density. It is clear, that CCMP is considered more reliable compared to other mobility prediction schemes as it is based mostly on live and not outdated information with the help of a cloud infrastructure.

\section{B. Future Work}

- Perform extensive simulations in order to demonstrate the validity of the proposed scheme.

- Investigate the effect of specific values for important algorithmic parameters such as time slot duration.

- Incorporate the use of machine learning in order to enhance the reliability of CCMP.

\section{REFERENCES}

[1] D. Astely, E. Dahlman, G. Fodor, S. Parkvall and J. Sachs, "LTE release 12 and beyond [Accepted From Open Call]," in IEEE Communications Magazine, vol. 51, no. 7, pp. 154-160, July 2013.

[2] J. Andrews, S. Buzzi, W. Choi, S. Hanly, A. Lozano, A. Soong, and J. Zhang, "What will 5G be?" IEEE J. Sel. Areas Commun., vol. 32, no. 6 , pp. 1065-1082, Jun. 2014.

[3] K. Sakaguchi, S. Sampei, H. Shimodaira, R. Rezagah, G. K. Tran and K. Araki, "Cloud cooperated heterogeneous cellular networks," Intelligent Signal Processing and Communications Systems (ISPACS), 2013 International Symposium on, Naha, 2013, pp. 787-791.

[4] K. Chen and R. Duan, "C-RAN: the road towards green RAN," White Paper, ver. 2, 2011.

[5] J. Zhang, A. Beletchi, Y. Yi, and H. Zhuang, "Capacity performance of millimeter wave heterogeneous networks at $28 \mathrm{GHz} / 73 \mathrm{GHz}$," in Proc. Globecom Workshops (GC Wkshps), 2014, Dec. 2014, pp. 405-409.

[6] Y. Niu, Y. Li, D. Jin, L. Su, and A. V. Vasilakos, "A survey of millimeter wave (mmWave) communications for 5G: Opportunities and challenges," arXiv preprint arXiv:1502.07228, Feb. 2015.

[7] Kyocera, "Potential performance of range expansion in macro-pico deployment (r1-104355)," 3GPP TSG RAN WG1 Meeting-62, Aug. 2010 .

[8] N. Brahmi et al., "METIS project deliverable D4.1: Summary on preliminary trade-off investigations and first set of potential networklevelsolutions," September 2013. [Online]. Available: https://www.metis2020.com/wp-content/uploads/deliverables/METIS D4.1 v1.pdf

[9] A. Mohamed, O. Onireti, S. Hoseinitabatabae, M. Imran, A. Imran, and R. Tafazolli, "Mobility prediction for handover management in cellular networks with control/data separation," in Proc. of IEEE International Conference on Communications (ICC), June 2015.

[10] N. Amirrudin, S. Ariffin, N. Malik, and N. Ghazali, "User's mobility history-based mobility prediction in LTE femtocells network," in Proc. of IEEE International RF and Microwave Conference, December 2013, pp. $105-110$. 